\title{
Clinical observation and analysis on the significance of quick sequential organ failure assessment in 74 non-ICU patients with sepsis
}

\author{
Xiaoyun Zhang, Junli Deng, Juan Chen, Yuancheng Huang, Liang Wu \\ Department of Infectious Diseases, the Affiliated Tongji Hospital of Tongji Medical College, Huazhong University of Science and Technology, \\ Wuhan, China \\ Contributions: (I) Conception and design: L Wu, X Zhang; (II) Administrative support: X Zhang, J Deng; (III) Provision of study materials or patients: \\ J Chen, Y Huang; (IV) Collection and assembly of data: X Zhang, J Deng, J Chen, Y Huang; (V) Data analysis and interpretation: L Wu, X Zhang; (VI) \\ Manuscript writing: All authors; (VII) Final approval of manuscript: All authors. \\ Correspondence to: Liang Wu. Department of Infectious Diseases, the Affiliated Tongji Hospital of Tongji Medical College, Huazhong University of \\ Science and Technology, Wuhan 430030, China. Email: wutongji@hotmail.com.
}

\begin{abstract}
Background Sepsis is an important disease that endangers human health and is the main cause of death in ICU patients, which has been a focus of clinical treatment. This study aims to evaluate the significance of the readily available quick sequential organ failure assessment (qSOFA) score in clinical cases of sepsis.

Methods: A retrospective cross-sectional study of patients with sepsis treated in the Department of Infectious Diseases, the Affiliated Tongji Hospital of Tongji Medical College, Huazhong University of Science and Technology from January 2015 to December 2016 was conducted, and the patients were divided into a high-score group ( $\geq 2$ points) and the low-score group ( $<2$ points) according to the diagnostic criteria for sepsis (Sepsis 3.0). The differences in disease outcome and inflammatory indicators were compared between groups.
\end{abstract}

Results: A total of 74 patients with sepsis were included in this study. When the cutoff qSOFA score was 2 points, the patients in the high-score group showed a higher mortality rate $(71.43 \%)$, and the patients in the low-score group showed a higher improvement rate $(87.76 \%)$. The inflammatory indicators did not show statistically significant differences between the two groups.

Conclusions: The qSOFA score can better predict the prognoses of non-ICU patients with sepsis compared with traditional inflammatory indicators. Clinicians should raise their awareness about qSOFA and improving its accuracy.

Keywords: Non-ICU patients; sepsis; quick sequential organ failure assessment (qSOFA); inflammatory indicators

Submitted Oct 12, 2020. Accepted for publication Jan 17, 2021.

doi: 10.21037/apm-20-2310

View this article at: http://dx.doi.org/10.21037/apm-20-2310

\section{Introduction}

Sepsis is a dysfunctional reaction to infection in the host, which leads to life-threatening organ damage (1). It develops quickly, and has a poor prognosis associated with high morbidity and mortality (2). Sepsis is still diagnosed according to clinical manifestations, but because early sepsis has non-specific clinical manifestations, there are currently no prompt and reliable early warning indicators (3). Therefore, once patients not in the intensive care unit (non-ICU) have sepsis, their prognosis worsens (4). The quick sequential organ failure assessment ( $q S O F A)$ was put forward at the Third International Consensus Definitions for Sepsis and Septic Shock (Sepsis $3.0)$ in 2016 (5). It consists of three items with a total score of $0-3$ points: 1 point is recorded respectively for systolic blood pressure $\leq 100 \mathrm{mmHg}$, shortness of breath $\geq 22 / \mathrm{min}$, 
altered mental status) (6). If the patient has two or more items, suspected sepsis will be diagnosed. The qSOFA can be used as a fast bedside sepsis screening tool for non-ICU patients and a positive score will raise clinicians' alertness about sepsis and thus reduce its potential mortality $(7,8)$. No practical study of qSOFA in clinical cases has been conducted, so we retrospectively analyzed the qSOFA scores of patients with sepsis were from January 2015 to December 2016, and comprehensively analyzed and compared them with related biochemical indicators such as platelets (PLT), procalcitonin (PCT), C-reactive protein (CRP), serum ferritin (SF) and lactic acid to evaluate the significance of qSOFA in clinical cases.

We present the following article in accordance with the STROBE reporting checklist (available at http://dx.doi. org/10.21037/apm-20-2310).

\section{Methods}

\section{Case data}

The study subjects were 74 patients with sepsis treated in the Department of Infectious Diseases from January 2015 to December 2016. The mean age was $(49.68 \pm 16.30)$ years, and the group comprised 43 males $(58.11 \%)$ and 31 females $(41.89 \%)$. This study was approved by the medical ethics committee of the Affiliated Tongii Hospital of Tongji Medical College. All procedures performed in this study involving human participants were in accordance with the Declaration of Helsinki (as revised in 2013). Individual consent for this retrospective analysis was waived.

\section{Diagnostic criteria}

The selected patients accorded with the diagnostic criteria for sepsis published by the 2012 International Sepsis Definitions Conference.

\section{Obvervational indicators and methods}

Obvervational indicators mainly included age, sex, etiology, infection site, vital signs, qSOFA score, PLT, PCT, CRP, SF and prognosis (9).

\section{qSOFA scoring and grouping method}

Archived electronic medical records from January 2015 to December 2016 were retrieved to find sepsis cases. Next, the three indicators (consciousness, systolic pressure and breathing) were searched for in the nursing records, the 3 -item nursing recording sheets, progress notes, rescue records and death record. Specific values were not recorded, it was only necessary to estimate whether altered mental status, systolic blood pressure $\leq 100 \mathrm{mmHg}$ and respiratory rate $\geq 22 / \mathrm{min}$ occurred in the patient during hospitalization. Patients with $\geq 2$ items were included in the high-score group ( $\geq 2$ points), as were patients with firstly one of three items meeting the scoring criteria and then the 2 nd and $3 \mathrm{rd}$ items meeting the scoring criteria within $2 \mathrm{~h}$ were also included in the high-score group.

\section{Laboratory tests}

The most unusual values for PLT, CRP, PCT, SF and lactic acid were observed during hospitalization.

\section{Prognosis judgment}

Patients leaving hospital without cure who died within 1 week after discharge from hospital were included in the death group.

\section{Statistical methods}

All data were analyzed using SPSS18.0 statistical software. Count data are expressed as a percentage. Continuous variables were measured using Student's test, categorical variables were measured using the chi-square test, and a $\mathrm{P}<0.05$ was considered statistically significant.

\section{Results}

\section{General information}

Data for 74 patients were analyzed, comprising 37 patients with pulmonary infection, 13 with intestinal infection, 9 with abdominal infection, 4 with urinary tract infection, 3 with limb bone infection, 1 patient each with biliary infection, and skin infection, 3 with intracranial infection and 3 with pelvic infection. There were 27 cases of positive blood culture, including 7 cases of Escherichia coli, 6 of Staphylococcus aureus, 4 of Klebsiella pneumoniae, 3 of Acinetobacter baumannii, 3 of Enterococcus faecium and 1 case each of Bacillus gasoformans, Candida tropicalis, Streptococcus mitis, Pseudomonas aeruginosa, Streptococcus, Bacillus subtilis, and Staphylococcus epidermidis. 
Table 1 qSOFA scores and outcomes of 74 patients with sepsis

\begin{tabular}{lcccc}
\hline Score & Death & Improvement & Summation & Chi-square \\
\hline qSOFA $<2$ & 6 & 43 & 49 & 24.519 \\
qSOFA $\geq 2$ & 15 & 6 & 21 & $<0.001$ \\
Summation & 21 & 49 & 70 & \\
\hline
\end{tabular}

qSOFA, quick sequential organ failure assessment.

Table 2 Comparison of inflammatory indicators in 74 sepsis patients with different outcomes

\begin{tabular}{lccc}
\hline Index & Death & Improvement & P value \\
\hline PLT $\left(\times 10^{9} / \mathrm{L}\right)$ & $132.57 \pm 125.99$ & $138.86 \pm 137.53$ & 0.853 \\
CRP $(\mathrm{mg} / \mathrm{L})$ & $87.88 \pm 80.80$ & $116.54 \pm 87.76$ & 0.197 \\
Lactic acid $(\mathrm{mmol} / \mathrm{L})$ & $2.79 \pm 1.95$ & $4.40 \pm 2.74$ & 0.102 \\
PCT $(\mathrm{ng} / \mathrm{L})^{*}$ & $0.13 \pm 0.83$ & $0.44 \pm 0.98$ & 0.178 \\
SF $(\mathrm{ng} / \mathrm{mL})^{*}$ & $3.35 \pm 0.61$ & $3.50 \pm 0.76$ & 0.451 \\
\hline
\end{tabular}

*, data did not meet the normal distribution, so log conversion was performed. CRP, C-reactive protein; PCT, procalcitonin; PLT, platelets; SF, serum ferritin.

Table 3 Comparison of inflammatory indicators in 74 sepsis patients with positive qSOFA score

\begin{tabular}{lccc}
\hline Index & qSOFA $<2$ & qSOFA $\geq 2$ & P value \\
\hline PLT $\left(\times 10^{9} / \mathrm{L}\right)$ & $91.81 \pm 100.59$ & $152.73 \pm 135.70$ & 0.069 \\
CRP $(\mathrm{mg} / \mathrm{L})$ & $95.52 \pm 79.82$ & $96.46 \pm 85.43$ & 0.966 \\
Lactic acid $(\mathrm{mmol} / \mathrm{L})$ & $4.75 \pm 2.78$ & $2.83 \pm 2.00$ & 0.065 \\
PCT $(\mathrm{ng} / \mathrm{L})^{*}$ & $0.23 \pm 0.86$ & $0.21 \pm 0.89$ & 0.934 \\
SF $(\mathrm{ng} / \mathrm{mL})^{*}$ & $3.77 \pm 0.66$ & $3.24 \pm 0.59$ & 0.003 \\
\hline
\end{tabular}

*, data did not meet the normal distribution, so log conversion was performed. CRP, C-reactive protein; PCT, procalcitonin; PLT, platelets; qSOFA, quick sequential organ failure assessment; SF, serum ferritin.

\section{qSOFA scores and outcomes}

We conducted a stratification analysis of the qSOFA scores of the 74 patients with sepsis (Table 1); those with qSOFA $\geq 2$ showed a higher mortality $(15 / 21,71.43 \%)$, whereas those with $\mathrm{qSOFA}<2$ showed a higher rate of improvement (43/49, 87.76\%).

\section{Comparison with inflammatory indicators}

We conducted a stratification analysis of the clinical outcomes of 74 patients with sepsis according to death or improvement (Table 2), and no significant differences in PLT, CRP, lactic acid, PCT and SF were observed.

\section{Comparison of qSOFA score for inflammatory indexes}

The 74 patients with sepsis were divided into two groups according to their qSOFA scores (Table 3). SF, PLT, CRP, lactate and PCT showed no significant differences.

\section{Discussion}

Sepsis is an important disease that endangers human health and is the main cause of death in ICU patients, which has been a focus of clinical treatment (10). At present, a variety of assessment systems are used to diagnose sepsis, including the sequential organ failure assessment (SOFA), diagnostic criteria for systemic inflammatory response syndrome 
(SIRS), logistic organ dysfunction score (LODS) and qSOFA (11). These evaluation systems for prognostication of sepsis patients in the ICU and non-ICU wards are currently an international research hotspot (12). In addition, inflammation-related indicators, including PLT, CRP, lactic acid, PCT and SF, are used for guiding clinicians in determining the condition and prognosis of sepsis patients.

\section{Significance of qSOFA score in non-ICU patients with sepsis}

It is difficult for non-ICU clinicians to timely and accurately perform complex qSOFA and continuous dynamic monitoring. The main reason for this is that the condition of patients with sepsis is often hidden and progresses rapidly. Non-ICU clinicians may have less contact with critical patients than ICU clinicians, and in non-ICU clinical work, all aspects of constant monitoring measures are worse than ICU clinical work. qSOFA needs no laboratory tests, its cost is lower, and quick and repeat assessment can be performed. A recent clinical trial cohort from Europe and the USA included 148,907 patients with suspected infection, among whom there were 6,347 (4\%) deaths. There were 7,932 patients with suspected infection in the cohort of ICU patients, among whom 1,289 (16\%) died, and the predictive efficacy of SIRS and qSOFA for the in-hospital mortality rate were inferior to that of SOFA or LODS. In the cohort of non-ICU patients, there were 66,522 patients with suspected infection and 1,886 deaths $(3 \%)$, and the predictive efficacy of qSOFA was higher than that of SOFA and SIRS. Compared with patients with qSOFA score $<2$, patients with qSOFA score $\geq 2$ had an inpatient mortality risk 3-14-fold higher than baseline (13). Our study data also showed similar results.

Another study from the German CAPNETZ multicenter database showed that the qSOFA score had a better predictive effect on the prognosis of new communityacquired pneumonia (CAP) sepsis (14). A domestic study analyzed the incidence rate of the poorest prognosis in 1,641 patients with CAP in China, and the area under the curve for the qSOFA predicting the mortality rate was 0.66 , suggesting that the qSOFA had a good predictive effect for the prognosis of CAP (15). Therefore, it is necessary for clinicians to assess the respiratory rate, blood pressure and mental status while also focusing on results of PLT, CRP, PCT, SF, and lactic acid for each patient who may be infected, especially in general wards, which will help clinicians to quickly observe early warnings and assess the condition of non-ICU patients with suspected infection, in order to implement early or strengthen the intervention and thereby improve the prognosis of patients with sepsis.

\section{Necessity for non-ICU clinicians to improve their awareness of qSOFA and its accuracy}

The condition of non-ICU patients with sepsis is often hidden and progresses rapidly, so a timely and accurate assessment is very important for them, not only to improve the success rate of rescue, but also to help physicians alert the patient's family members on disease prognosis to reduce the occurrence of medical disputes. Unlike the SOFA of ICU patients with sepsis, the scoring indicators for the qSOFA require detailed observation by clinicians. In routine care of infectious disease patients, the frequency of observation of vital signs of fever patients is 4 times/day, and that frequency is increased to once every $4 \mathrm{~h}$ when the body temperature exceeds $39.0^{\circ} \mathrm{C}$. In the case notes of the 74 study patients, the indicators with the highest frequency in the early stage were body temperature, and the pulse and respiration rates. In the process of temperature decreasing, consciousness and blood pressure were observed. When the patient had an obvious abnormality in one of the indicators (i.e., consciousness, systolic blood pressure and breathing), ECG monitoring was instituted, and the observation frequency increased to once every $30-60 \mathrm{~min}$, and even higher when the patient was critically ill.

In the early stage of sepsis ,the envelope glycoprotein in the surface of endothelial cells is damaged, endothelial permeability is increased and the spaces between endothelial cells are expanded due to inflammatory response. Plasma leaks out the circulatory system, causing a drop in blood pressure (16). In routine clinical nursing, no specific requirement on the frequency of blood pressure measurement is made for patients with fever, so the need for continuous observation of blood pressure is strengthened for patients with suspected infection in general wards (17). On the one hand, the observed value of systolic blood pressure is increased by $10 \mathrm{mmHg}$ compared with blood pressure during shock in accordance with the standard qSOFA score. On the other hand, for patients with high basal blood pressure, attention should be paid to the systolic blood pressure variety ratio. In the study by Zhang and $\mathrm{Li}$ (18), they found that the blood pressure variety ratio of the patient positively correlated with the severity of sepsis, and the systolic blood pressure variety ratio can affect the severity of sepsis, so timely effective measures should be 
taken for sepsis patients with continuously reduced systolic blood pressure (19). Blood pressure measurement was recorded in the case notes of the 74 patients mostly because they were sweating after the high temperature was lowered. Measurement frequency was significantly increased if the systolic blood pressure $\leq 90 \mathrm{mmHg}$, and ECG monitoring was also used.

The study of Xie et al. (20) showed that for shock patients, the value of monitoring blood pressure using an electronic sphygmomanometer was higher than using invasive blood pressure monitor or the mercury sphygmomanometer. In addition, the lower the blood pressure, the greater the difference between the monitoring values, which indicates that when the systolic blood pressure is $\leq 100 \mathrm{mmHg}$, attention should be paid to fixing the limb and using a mercury sphygmomanometer to avoid the measured value of blood pressure being affected by factors such as physical activity, muscle spasm and clothing. In addition, if the systolic blood pressure is $\leq 100 \mathrm{mmHg}$ during clinical observation, then observation of changes in respiratory rate should be immediately enhanced. The respiratory rate is a reliable and sensitive indicator of acute respiratory insufficiency (21). The measurement time should be not less than $60 \mathrm{~s}$, in order to guide clinicians about the possible existence of respiratory function abnormalities in a timely manner. If the patient's systolic blood pressure and breathing both meet the scoring criteria, this situation should be immediately reported to the physicians to improve the rescue time and the effect of treatment.

In addition to QSOFA, early warning score (MEWS), simplified sequential organ failure score (sSOFA), systemic inflammatory response syndrome (SIRS) score and lactate level can also be used as bedside screening tools for patients with sepsis. The advantages of qSOFA scoring system are: simple and easy to operate, comprehensive scoring of patients' vital signs, rapid and repeated evaluation at the bedside, digitization and visualization of the disease, and superior in the immediacy, simplicity, rapidity and practicability of disease assessment. Early and rapid assessment of the patient's condition, according to the risk degree of effective monitoring and treatment is particularly important. The non-intensive care unit lacks corresponding experience in the diagnosis of sepsis and lacks powerful monitoring equipment and diagnostic equipment. Because the doctors in the non-intensive care unit have fewer patients in the diagnosis of sepsis than the doctors in the intensive care unit, the alertness of doctors in the nonintensive care unit in the diagnosis of sepsis is lower than that in the intensive care unit. In terms of treatment, there are not many patients with sepsis contacted by doctors in the non-intensive care unit. Therefore, there is also a lack of corresponding experience in treatment, and the process of effective treatment will be slow. Doctors in non-intensive care units should timely follow up and learn the guidelines for the diagnosis and treatment of sepsis, constantly improve their own quality and the level of knowledge and skills, and even go deep into the intensive care unit to learn the knowledge of sepsis diagnosis and treatment.

\section{Acknowledgments}

Funding: None.

\section{Footnote}

Reporting Checklist: The authors have completed the STROBE reporting checklist. Available at http://dx.doi. org/10.21037/apm-20-2310

Data Sharing Statement: Available at http://dx.doi. org/10.21037/apm-20-2310

Conflicts of Interest: All authors have completed the ICMJE uniform disclosure form (available at http://dx.doi. org/10.21037/apm-20-2310). The authors have no conflicts of interest to declare.

Ethical Statement: The authors are accountable for all aspects of the work in ensuring that questions related to the accuracy or integrity of any part of the work are appropriately investigated and resolved. This study was approved by the medical ethics committee of the Affiliated Tongji Hospital of Tongji Medical College. All procedures performed in this study involving human participants were in accordance with the Declaration of Helsinki (as revised in 2013). Individual consent for this retrospective analysis was waived.

Open Access Statement: This is an Open Access article distributed in accordance with the Creative Commons Attribution-NonCommercial-NoDerivs 4.0 International License (CC BY-NC-ND 4.0), which permits the noncommercial replication and distribution of the article with the strict proviso that no changes or edits are made and the original work is properly cited (including links to both the formal publication through the relevant DOI and the license). 
See: https://creativecommons.org/licenses/by-nc-nd/4.0/.

\section{References}

1. Cecconi M, Evans L, Levy M, et al. Sepsis and septic shock. Lancet 2018;392:75-87.

2. Wu W, Fan $Z$, Yao C, et al. Platelet maximum aggregation rate serves as a marker in diagnosis and prognosis in patients with sepsis. Ann Palliat Med 2020;9:847-57.

3. Huang M, Cai S, Su J. The Pathogenesis of Sepsis and Potential Therapeutic Targets. Int J Mol Sci 2019;20:5376.

4. Chang EY, Stevens ES, Bakinde N. Sepsis in Non-ICU Patients: A Call for Guideline Reassessment. Crit Care Med 2019;47:e266.

5. Singer M, Deutschman CS, Seymour CW, et al. The Third International Consensus Definitions for Sepsis and Septic Shock (Sepsis-3). JAMA 2016;315:801-10.

6. Charitos IA, Topi S, Castellaneta F, et al. Current Issues and Perspectives in Patients with Possible Sepsis at Emergency Departments. Antibiotics (Basel) 2019;8:56.

7. Franchini S, Scarallo L, Carlucci M, et al. SIRS or qSOFA? Is that the question? Clinical and methodological observations from a meta-analysis and critical review on the prognostication of patients with suspected sepsis outside the ICU. Intern Emerg Med 2019;14:593-602.

8. Seymour CW, Liu VX, Iwashyna TJ, et al. Assessment of Clinical Criteria for Sepsis: For the Third International Consensus Definitions for Sepsis and Septic Shock (Sepsis-3). JAMA 2016;315:762-74.

9. Huang Q, Sun Y, Luo L, et al. A new warning scoring system establishment for prediction of sepsis in patients with trauma in intensive care unit. Zhonghua Wei Zhong Bing Ji Jiu Yi Xue 2019;31:422-7.

10. Zhang Z, Bokhari F, Guo Y, et al. Prolonged length of stay in the emergency department and increased risk of hospital mortality in patients with sepsis requiring ICU admission. Emerg Med J 2019;36:82-7.

11. Goulden R, Hoyle MC, Monis J, et al. qSOFA, SIRS and NEWS for predicting inhospital mortality and ICU

Cite this article as: Zhang X, Deng J, Chen J, Huang Y, Wu L. Clinical observation and analysis on the significance of quick sequential organ failure assessment in 74 non-ICU patients with sepsis. Ann Palliat Med 2021;10(1):244-249. doi: 10.21037/ apm-20-2310 admission in emergency admissions treated as sepsis. Emerg Med J 2018;35:345-9.

12. Mukhopadhyay S, Taylor JA, Von Kohorn I, et al. Variation in Sepsis Evaluation Across a National Network of Nurseries. Pediatrics 2017;139:e20162845.

13. Raith EP, Udy AA, Bailey M, et al. Prognostic Accuracy of the SOFA Score, SIRS Criteria, and qSOFA Score for In-Hospital Mortality Among Adults With Suspected Infection Admitted to the Intensive Care Unit. JAMA 2017;317:290-300.

14. Forstner C, Rohde G, Rupp J, et al. Community-acquired Haemophilus influenzae pneumonia--New insights from the CAPNETZ study. J Infect 2016;72:554-63.

15. Cao B, Huang Y, She DY. Diagnosis and treatment of community-acquired pneumonia in adults: 2016 clinical practice guidelines by the Chinese Thoracic Society, Chinese Medical Association. Clin Respir J 2018;12:1320-60.

16. Iampietro M, Younan $\mathrm{P}$, Nishida A, et al. Ebola virus glycoprotein directly triggers $\mathrm{T}$ lymphocyte death despite of the lack of infection. PLoS Pathog 2017;13:e1006397.

17. Qiu MS, Yin HY. Advances in the research of sepsisrelated stress response. Zhonghua Shao Shang Za Zhi 2016;32:765-8.

18. Zhang ZR, Li Q. Relationship between blood pressure variability rate and extent of critically in ill patients with sepsis. China Modern Doctor 2015;000:4-6,10.

19. Davidson S, Pretty C, Balmer J, et al. Blood pressure waveform contour analysis for assessing peripheral resistance changes in sepsis. Biomed Eng Online 2018;17:171.

20. Xie YM, Wang FZ, Huang HC. Comparison of three BP Measurement methods in shock patients. Journal of Nursing Science 2008;23:3-4.

21. Gu X, Zhou F, Wang Y, et al. Respiratory viral sepsis: epidemiology, pathophysiology, diagnosis and treatment. Eur Respir Rev 2020;29:200038.

(English Language Editor: K. Brown) 